\title{
MÉTODO CANGURU: CONHECIMENTOS E PRÁTICAS DA EQUIPE MULTIPROFISSIONAL
}

KANGAROO METHOD: KNOWLEDGE AND PRACTICES OF THE MULTIPROFESSIONAL TEAM

\author{
Ana Maria de Souza Matozo ${ }^{1} *$ Mayara Carolina Cañedo ${ }^{2} *$ Cristina Brandt Nunes $^{3} *$ Thiago Inácio $^{2}$ \\ Barros Lopes ${ }^{4}$
}

\begin{abstract}
RESUMO
Objetivo: Analisar o conhecimento e práticas dos profissionais de saúde que atuam na linha pediátrica de um hospital de ensino de Campo Grande/Mato Grosso do Sul sobre o Método Canguru. Métodos: Estudo descritivo e transversal realizado com dados primários, por meio de questionário aplicado de agosto a setembro de 2020 e na primeira quinzena de janeiro de 2021, com 98 profissionais de saúde da linha pediátrica. Os dados coletados foram armazenados e calculados por meio do programa Microsoft Office Excel. Resultados: A maioria foi do sexo feminino, declarada de cor branca e escolaridade de ensino superior com pós-graduação. Quanto aos aspectos profissionais, obteve a predominância de técnicos de enfermagem e tempo de serviço na instituição de aproximadamente 12 anos. A pesquisa demonstrou um maior percentual de respostas adequadas sobre o método, a Posição Canguru e sobre os seus benefícios nas explicações dos profissionais da saúde que tinham o curso do Método Canguru, mais tempo de serviço na linha pediátrica e que atuavam na unidade neonatal. Além disso, a maioria tem conhecimento sobre o método e em quantas etapas se divide. Entretanto, alguns participantes têm a opinião equivocada de que o Método Canguru se restringe à Posição Canguru. Conclusões: $\mathrm{O}$ estudo possibilitou a reflexão sobre o entendimento do método pelos profissionais da saúde que realizam assistência aos recém-nascidos e seus familiares. Tal fato pode contribuir para o aperfeiçoamento assistencial, além de incentivar os gestores de saúde na inserção da educação permanente e continuada dos profissionais da saúde sobre o Método Canguru.
\end{abstract}

Palavras-chave: Método Canguru; Equipe de Assistência ao Paciente; Recém-Nascido; Enfermagem Neonatal; Serviços de Saúde da Criança.

\begin{abstract}
Objective: To analyze the knowledge and practices of health professionals working in the pediatric line of a teaching hospital in Campo Grande/Mato Grosso do Sul about the Kangaroo Method. Methods: Descriptive and cross-sectional study carried out with primary data, through a questionnaire applied from August to September 2020 and in the first half of January 2021, with 98 pediatric healthcare professionals. The collected data were stored and calculated using the Microsoft Office Excel program. Results: The majority were female, declared to be white and had completed higher education with a postgraduate degree. As for professional aspects, there was a predominance of nursing technicians and length of service at the institution of approximately 12 years. The survey showed a higher percentage of adequate answers about the method, the Kangaroo Position and about its benefits in the explanations of health professionals who had taken the Kangaroo Method course, more time in the pediatric line and who worked in the neonatal unit. In addition, most are knowledgeable about the method and how many steps it is divided into. However, some participants are of the mistaken opinion that the Kangaroo Method is restricted to the Kangaroo Position. Conclusion: The study allowed for reflection on the understanding of the method by health professionals who provide care to newborns and their families. This fact can contribute to the improvement of care, in addition to encouraging health managers in the insertion of permanent and continuing education for health professionals about the Kangaroo Method.
\end{abstract}

Keywords: Kangaroo-Mother Care Method; Patient Care Team; Infant, Newborn; Neonatal Nursing; Child Health Services.

\footnotetext{
${ }^{1}$ Pós-graduada pelo Programa de Residência Multiprofissional Integrada em Saúde Materno Infantil pelo Hospital Regional de Mato Grosso do Sul/Anhanguera Uniderp. Enfermeira na unidade neonatal da Associação de Amparo a maternidade e a infância, Campo Grande, Mato Grosso do Sul, Brasil. ORCID: https://orcid.org/0000-0001-6499-1278

${ }^{2}$ Mestre em enfermagem pela Universidade Federal de Mato Grosso do Sul. Enfermeira do Núcleo Regional de Saúde de Dourados, Mato Grosso do Sul, Brasil. ORCID: http://orcid.org/0000-0002-7232-1431

${ }^{3}$ Doutora em Ciências pela Universidade Federal de São Paulo. Professora Associada (aposentada) da Universidade Federal de Mato Grosso do Sul, Brasil. ORCID: http://orcid.org/0000-0003-2411-0717

${ }^{4}$ Doutor em Química Orgânica pela Universidade Estadual de Campinas, Brasil. ORCID: https://orcid.org/0000-0002-5913-1289
} 


\section{INTRODUÇÃO}

O nascimento de um recém-nascido pré-termo de baixo peso (RNPTBP) causa impacto emocional nas famílias, devido à necessidade de hospitalização e a permanência em incubadoras (1). Ademais, pesquisa realizada em um hospital regional do Piauí, Brasil, com 125 prontuários de recémnascidos (RN) hospitalizados em uma Unidade de Terapia Intensiva Neonatal (UTIN) demonstrou que os partos de bebês pré-termo, ocorreram ainda que as gestantes tenham cumprindo com o número de consultas de pré-natal recomendadas pelo Ministério da Saúde ${ }^{(2)}$.

Diante do exposto, torna-se relevante a criação de redes de atenção à saúde (RAS) efetiva e regionalizada para a atenção neonatal. No ano de 1979, surgiu na Colômbia a "Mãe Canguru" com o intuito de colocar o RNPTBP em contato pele a pele contínuo contra o tórax materno e assim melhorar a assistência prestada. No Brasil, o Método Canguru (MC) ganhou visibilidade nacional com o Instituto Materno Infantil de Pernambuco, atualmente Instituto de Medicina Integral Professor Fernando Figueira (IMIP), que foi um dos finalistas do concurso de projetos sociais patrocinado pelo Banco Nacional de Desenvolvimento Econômico e Social (BNDES), com o nome “Enfermaria Mãe Canguru”. A partir de então, diversos hospitais brasileiros adotaram o novo modelo de assistência ao recém-nascido prétermo $\left(\right.$ RNPT) ${ }^{(3)}$.
Com o intuito de melhorar a qualidade da atenção à saúde da gestante, do RNPTBP e de sua família, o MC no Brasil é dividido em três etapas. A primeira é desenvolvida no pré-natal e durante o nascimento acompanhado da internação do RN na UTIN e/ou na Unidade de Cuidado Intermediário Neonatal Convencional (UCINCo). A segunda é a participação dos pais nos cuidados neonatais na Unidade de Cuidado Intermediário Neonatal Canguru (UCINCa); e a última etapa se dá no domicílio da família, com acompanhamentos compartilhados entre as consultas ambulatoriais no hospital de origem e a Atenção Primária à Saúde (APS) ${ }^{(4,5)}$.

Esse método traz benefícios para o RNPTBP, pais/familiares e para os profissionais, e melhora o controle térmico, proporciona o aleitamento materno (AM) mais efetivo, o ganho de peso, a redução do estresse, e outros fatores. Para os pais/familiares, trata-se do momento de estabelecer vínculo entre o trinômio mãefilho-pai e diminuir a insegurança. Os profissionais da saúde, por sua vez, desempenham o papel importante de orientar, incentivar as famílias nos cuidados com os bebês, estabelecer vínculo e prestar o cuidado individualizado e humanizado em todos os $\mathrm{RN}^{(4,5)}$.

Assim, a equipe de saúde que atua de acordo com os paradigmas do MC possui mais envolvimento com a família, ajuda no enfrentamento menos traumático da 
internação, realiza melhor orientações nos cuidados com o RNPT, além de identificar a melhora na evolução clínica do RN. A equipe multiprofissional tem um papel facilitador e motivador para a efetivação do $\mathrm{MC}$, promovendo a sua implantação nas unidades de saúde ${ }^{(6)}$.

Pesquisa realizada com 22 profissionais de saúde de um Centro de Terapia Intensiva Neonatal e Pediátrica, cujo objetivo era conhecer os benefícios e os desafios vivenciados por eles acerca do MC, demonstrou que cada profissional e instituição com suas particularidades únicas influenciam na implementação do método. Além disso, os autores reforçam que o MC exige comprometimento da gestão e a perspicácia do serviço para analisar o seu ambiente, aprimorar os recursos humanos e adequá-lo, em prol do RNPTBP e de sua família ${ }^{(7)}$.

Diante do exposto, esta pesquisa se justifica pela escassez de estudos envolvendo as percepções e os conhecimentos da equipe multidisciplinar sobre o MC ${ }^{(8)}$. Assim, com base na literatura, foi elaborada a seguinte questão de pesquisa: Qual o conhecimento e práticas da equipe de saúde da linha pediátrica sobre o MC?

O objetivo deste estudo, portanto, foi analisar o conhecimento e práticas dos profissionais de saúde que atuam na linha pediátrica de um hospital de ensino de Campo Grande/Mato Grosso do Sul (MS) sobre o MC.

\section{MÉTODOS}

Trata-se de uma pesquisa exploratória transversal, com abordagem metodológica quantitativa. $\mathrm{O}$ estudo foi realizado com os profissionais que atuam na linha pediátrica de um hospital de ensino localizado em Campo Grande - MS, que atende pacientes do Sistema Único de Saúde (SUS). Além disso, desde 2012, o hospital é credenciado como referência estadual para o MC e também como Iniciativa Hospital Amigo da Criança (IHAC).

A população foi constituída pela equipe multiprofissional da linha pediátrica do referido hospital. Essa equipe é formada por enfermeiros (as), técnicos (as) de enfermagem, técnicos (as) de lactário, auxiliar de enfermagem, médicos (as) (pediatra; neonatologista e intensivista pediátrico), fisioterapeutas, terapeutas ocupacionais, assistentes sociais, fonoaudiólogas e nutricionistas, totalizando 179 funcionários. Foram incluídos na pesquisa profissionais da linha pediátrica que atuavam nos três turnos de trabalho com, no mínimo, seis meses de atuação e foram excluídos 13 profissionais que se encontravam de licença médica e seis que estavam de férias, restando, portanto, 160 profissionais elegíveis.

A amostra do presente estudo foi constituída por 98 profissionais, com base na técnica amostral probabilística simples com nível de confiança de $95 \%$ e erro amostral de $5 \%$. A seleção foi realizada por conveniência. As pesquisadoras realizaram contato prévio 
pessoalmente com os participantes. Destes, seis profissionais se recusaram a responder ao questionário, assim, outros seis que não haviam sido abordados ainda e que trabalhavam na linha pediátrica foram convidados para completar a amostra.

Esta investigação utilizou dados primários por meio de questionário próprio desenvolvido pelas autoras após a realização da revisão bibliográfica. Além disso, para testar a adequação do instrumento aos objetivos desta pesquisa, as pesquisadoras aplicaram um pré-teste com um participante no mês de agosto de 2020 e esse questionário faz parte da pesquisa.

A coleta de dados foi realizada de agosto a setembro de 2020 e, para alcançar o cálculo amostral, os dados foram coletados também na primeira quinzena de janeiro de 2021. Por causa da pandemia pelo Coronavírus, os profissionais da linha pediátrica foram remanejados para a linha do paciente crítico adulto, sendo necessária essa prorrogação no tempo de coleta. Os questionários foram aplicados em horários previamente agendados com os participantes a fim de não prejudicar o desenvolvimento de suas atividades profissionais. As variáveis quantitativas foram apresentadas de forma descritiva por meio de médias, porcentagens, desvio-padrão e probabilidade de significância. Todos os cálculos foram realizados utilizando o programa Microsoft Office Excel.
Este estudo foi submetido à Diretoria de Ensino, Pesquisa e Qualidade Institucional do hospital de ensino e ao Comitê de Ética em Pesquisa com Seres Humanos da Universidade para o Desenvolvimento do Estado e da Região do Pantanal (CEP/UNIDERP). O projeto foi aprovado pelo CEP/UNIDERP com o protocolo CAAE $\mathrm{n}^{\circ} \quad 34288620.8 .0000 .5161, \quad$ parecer $\mathrm{n}^{\circ}$ 4.179.030 e emenda 18/12/2020 parecer $n^{\circ}$ 4.476.263. A coleta de dados foi iniciada após a autorização dos participantes, por meio do Termo de Consentimento Livre e Esclarecido. Para assegurar os direitos dos participantes, foram observados todos os itens presentes na Resolução do Conselho Nacional de Saúde 466/12.

\section{RESULTADOS}

O estudo foi realizado com 98 profissionais da saúde com idade média $42,8 \pm 7,8$ anos (média \pm desvio padrão) e renda média em salários-mínimos de 10,0 $\pm 12,0$ (média \pm desvio padrão). A maioria foi do sexo feminino ( $\mathrm{n}=90 ; 92 \%)$, declarada de cor branca $(\mathrm{n}=55 ; 56 \%)$ e escolaridade de ensino superior com pós-graduação (especialização) ( $\mathrm{n}=35 ; 36 \%)$. Quanto aos aspectos profissionais, o estudo obteve a predominância de técnicos de enfermagem $\mathrm{n}=40 \quad(40,8 \%)$ e tempo de serviço na instituição de 12,1 $\pm 5,7$ anos (média \pm desvio padrão). Quanto ao tipo de vínculo com a instituição, 82 profissionais $(84 \%)$ são estatutários, com tempo de serviço médio no 
setor de 11,4 anos; e $52(53 \%)$ atuam na Unidade Neonatal (UCINCa/UCINCo/UTIN). participantes quanto às características sociodemográficas e formação profissional.

A Tabela 1 apresenta a distribuição dos

Tabela 1 - Distribuição das informações sociodemográficas dos profissionais da linha pediátrica. Campo Grande - MS, 2020/2021.

\begin{tabular}{|c|c|c|}
\hline \multicolumn{2}{|l|}{ Variáveis } & Média \pm DP \\
\hline \multicolumn{2}{|c|}{ Idade (anos) } & $42,8 \pm 7,8$ \\
\hline \multicolumn{2}{|c|}{ Tempo de serviço (anos) } & $12,1 \pm 5,7$ \\
\hline \multicolumn{2}{|c|}{ Renda (salários mínimos) } & $10,0 \pm 12,0$ \\
\hline \multicolumn{2}{|l|}{ Variáveis } & $\mathbf{n}(\%)$ \\
\hline & Amarela & $4(4)$ \\
\hline & Branca & $55(56)$ \\
\hline \multirow[t]{3}{*}{ Raça/Cor } & Parda & $33(34)$ \\
\hline & Preta & 4(4) \\
\hline & Nãoinformada & 2(2) \\
\hline \multirow{6}{*}{ Sexo } & Feminino & $90(92)$ \\
\hline & Masculino & $8(8)$ \\
\hline & AssistenteSocial & $2(2)$ \\
\hline & AuxiliardeEnfermagem & $6(6)$ \\
\hline & Enfermeira & $12(12)$ \\
\hline & Fisioterapeuta & $10(10)$ \\
\hline \multirow{8}{*}{ Profissão } & Fonoaudióloga & 3(3) \\
\hline & Médico & $17(17)$ \\
\hline & Nutricionista & $3(3,1)$ \\
\hline & TécnicodeEnfermagem & $40(40,8)$ \\
\hline & TécnicodeLactário & 3(3) \\
\hline & TerapeutaOcupacional & $2(2)$ \\
\hline & MédioCompleto & $26(26)$ \\
\hline & SuperiorIncompleto & $5(6)$ \\
\hline \multirow{4}{*}{ Escolaridade } & SuperiorCompleto & $10(10)$ \\
\hline & Especialização & $35(36)$ \\
\hline & Residência & $16(16)$ \\
\hline & Mestrado & $6(6)$ \\
\hline \multicolumn{2}{|l|}{ Total } & $98(100)$ \\
\hline
\end{tabular}

Legenda: n, número de servidores; DP, desvio padrão.

Fonte: Elaboração própria.

Em relação à realização do curso do MC, 69 (70\%) profissionais relataram tê-lo feito e 29 (30\%), não. Sobre o número de vezes que os profissionais o realizaram, 33
(34\%) não souberam ou não responderam; 18 $(18 \%)$ o fizeram mais de três vezes; cinco (5\%), três vezes; 22 (22\%), duas vezes; e 20 (20\%) somente uma vez. 
Quanto à data do último curso realizado pelos profissionais, 56 (57\%) não souberam ou não responderam; 15 (15\%) fizeram o último curso em 2017; oito (8\%), antes de 2017; e seis (6\%), em 2018. Além desses dados, 58 (59\%) profissionais responderam que não lhes foi perguntado pelo hospital do estudo se já tinham o curso de MC quando começaram a trabalhar na linha pediátrica; somente nove (9\%) afirmaram que foram indagados. Vinte (20\%) entraram na instituição antes de 2012, período em que o método não havia sido implementado, e 11 (11\%) não se lembram.

Com relação à instituição em que os profissionais receberam a capacitação do $\mathrm{MC}$, 53 deles o realizaram no hospital de estudo, 19 indicaram outras intuições e seis não informaram o local. Quanto ao tipo de curso, 64 participantes realizaram o de $\mathrm{MC}$ Hospitalar; 49, de MC APS; sete, de tutor do MC APS; e somente dois, para tutor do MC Hospitalar.

Quando os profissionais foram questionados sobre o conhecimento que tinham sobre o MC, 48 (50\%) das respostas estavam relacionadas à assistência humanizada ao RNPTBP e sua família no ambiente hospitalar, visando os aspectos biopsicossociais e 39 (39\%) descreveram-no como sendo contato pele a pele do $\mathrm{RN}$ com a mãe e/ou pai. Conforme a opinião dos pesquisados sobre o que é a Posição Canguru (PC), 54 (55\%) descreveram-no como: "contato pele a pele do RNPTBP com seus pais, junto ao peito na posição vertical”; 35 (36\%), "contato pele a pele do RNPTBP com sua mãe"; e nove (9\%) não responderam ou não sabem.

Nas respostas sobre os cuidados prestados ao RNPTBP e sua família de acordo com o MC, 34 responderam: "Orientações e acolhimento aos pais sobre os cuidados ao RN”; 22, "Diminuição dos estímulos ambientais (ruídos, luminosidade, temperatura); dez, "Estimular os pais na realização da PC"; nove, "Realização de medidas para controle de estresse e dor (farmacológica e não farmacológica)"; oito, “Orientações quanto à prevenção de infecção hospitalar e higienização das mãos"; e 25 não responderam ou não sabem.

Quanto aos benefícios do MC, os três mais descritos, por ordem, pelos profissionais da saúde foram: "Aumenta o vínculo familiar e bebê" $(\mathrm{n}=70), \quad$ "Estimula/facilita a amamentação" $(\mathrm{n}=40)$ e "Favorece o desenvolvimento neuropsicomotor do RN" $(\mathrm{n}=30)$, sendo observado que alguns participantes responderam mais de um item. Ademais, o estudo revelou diferença estatística $(p=0,001)$ entre os profissionais que realizaram o curso do $\mathrm{MC}$ e aqueles que não o realizaram, ao serem questionados sobre quem se beneficiava com o MC.

A Tabela 2 apresenta os resultados relativos às questões sobre o $\mathrm{MC}$, como conhecimento do método e PC, critérios para o RNPTBP ir para UCINCa e cuidados baseados na metodologia canguru, 
evidenciando que os profissionais que apresentaram tempo médio de serviço mais longo no setor e que declararam terem feito o curso sobre o MC foram os que responderam corretamente em quantas etapas se divide o MC 56 (81\%), sobre o momento correto de se iniciar o $\mathrm{MC} \quad 48$ (70\%) e $\mathrm{o}$ tempo recomendado para a realização da PC 30 (43\%). Dos 69 profissionais que fizeram o curso do MC, 56 souberam responder que o método se divide em três etapas, mas somente $11(20 \%)$ souberam explicar cada uma delas.

Com relação ao cuidado singular centrado no RN e sua família, 64 (65\%) dos profissionais já ofereceram assistência baseada no MC, ressaltando que dentre estes, $53(77 \%)$ possuem o curso de MC. No que diz respeito aos critérios para que o RNPTBP participe da segunda etapa do MC, dentre os participantes que têm curso do método, 59 (86\%) responderam que sabem as condições para que o RNPTBP vá para a UCINCa; porém apenas 12,9 (22\%) deles elencaram os critérios em sua totalidade. Por outro lado, 13 (45\%) dos participantes que declararam ter ciência dos critérios, mas não ter feito o curso do $\mathrm{MC}$, apenas 5,8 (15\%) responderam corretamente às perguntas sobre os critérios necessários para a transferência do bebê e sua família para a segunda etapa.

Tecendo um paralelo entre as respostas do questionário e os setores avaliados, nota-se que os profissionais da unidade neonatal possuem o maior índice de conhecimento relacionado ao $\mathrm{MC}$, podendo estar relacionado com a prática profissional, visto que são os profissionais que mais recepcionam, atende e cuida dos RNPTBP. Esse dado demonstra a necessidade de capacitações para a Unidade de Terapia Intensiva Pediátrica (UTIP), para a enfermaria de pediatria que atende o RNPTBP, e para o banco de leite, que atende a mãe do bebê prétermo. A Tabela 3 apresenta os resultados relativos às questões sobre o $\mathrm{MC}$ em função do setor de atuação. 
Tabela 2 - Conhecimentos dos profissionais da linha pediátrica sobre o Método Canguru em função da realização do curso e do tempo médio de serviço. Campo Grande - MS, 2020/2021.

\begin{tabular}{|c|c|c|c|c|c|c|}
\hline \multirow{2}{*}{\multicolumn{2}{|c|}{ Conhecimentos dos profissionais de saúde da linha pediátrica sobre o Método Canguru }} & \multicolumn{2}{|c|}{$\underline{\text { Recebeu capacitação }}$} & \multirow{3}{*}{$\frac{\mathbf{p}}{0,573}$} & \multirow{2}{*}{$\begin{array}{c}\text { Tempo } \\
\text { médio de } \\
\text { serviço } \\
\end{array}$} & \multirow{2}{*}{$\begin{array}{c}\text { Total } \\
(\mathbf{n}=98) \\
\mathbf{n}(\%)\end{array}$} \\
\hline & & $\begin{array}{l}\operatorname{Sim}(\mathbf{n}=69) \\
\mathbf{n}(\%)\end{array}$ & $\begin{array}{c}\text { Não (n }=29) \\
n(\%)\end{array}$ & & & \\
\hline \multirow{4}{*}{ Tipo de vínculo } & Celetista & $4(6)$ & $5(17)$ & & 2,5 & $9(9)$ \\
\hline & Estatutário & $64(93)$ & $18(62)$ & \multirow{6}{*}{0,198} & 9,8 & $82(84)$ \\
\hline & Outros & $1(1)$ & $5(17)$ & & 1,8 & $6(6)$ \\
\hline & Não respondeu & $0(0)$ & $1(3)$ & & 3,0 & $1(1)$ \\
\hline \multirow{5}{*}{ Tempo de serviço na linha pediátrica } & $<1$ ano & $4(6)$ & $6(21)$ & & 0,3 & $10(10)$ \\
\hline & $1<$ tempo $<3$ anos & $8(12)$ & $10(34)$ & & 1,4 & $18(18)$ \\
\hline & $3<$ tempo $<5$ anos & $8(12)$ & $1(3)$ & & 2,0 & $9(9)$ \\
\hline & $5<$ tempo $<10$ anos & $16(23)$ & $6(14)$ & \multirow{5}{*}{0,301} & 10,3 & $22(22)$ \\
\hline & $>10$ anos & $31(45)$ & $6(21)$ & & 22,1 & $37(38)$ \\
\hline \multirow{7}{*}{ Setor em que atua } & Não respondeu & $2(3)$ & $0(0)$ & & 0,0 & $2(2)$ \\
\hline & UTI Pediátrico & $13(19)$ & $6(21)$ & & 9,9 & $19(19)$ \\
\hline & $\begin{array}{l}\text { Unidade Neonatal } \\
\text { (UCINCa/UCINCo/UTI }\end{array}$ & & & & & \\
\hline & Neonatal) & $40(58)$ & $12(41)$ & & 10,0 & $52(53)$ \\
\hline & Pediatria & $13(19)$ & $9(31)$ & \multirow{4}{*}{0,506} & 13,2 & $22(22)$ \\
\hline & Banco de Leite & $3(4)$ & $2(7)$ & & 18,3 & $5(5)$ \\
\hline & Tem conhecimento & $56(81)$ & $14(48)$ & & 11,3 & $70(71)$ \\
\hline \multirow[t]{2}{*}{ Conhecimento sobre o número de etapas do Método Canguru } & Não tem conhecimento & $7(10)$ & $3(10)$ & & 5,7 & $10(10)$ \\
\hline & Não sabe ou não respondeu & $6(9)$ & $12(41)$ & \multirow{3}{*}{0,362} & 10,1 & $18(18)$ \\
\hline \multirow[t]{2}{*}{ Conhecimento sobre o momento correto de se iniciar o Método Canguru } & Tem conhecimento & $48(70)$ & $10(34)$ & & 11,6 & $58(59)$ \\
\hline & Não tem conhecimento & $21(30)$ & 19(66) & & 8,0 & $40(41)$ \\
\hline \multirow{3}{*}{$\begin{array}{l}\text { Conhecimento sobre tempo recomendado para a realização da Posição } \\
\text { Canguru }\end{array}$} & Correto & $30(43)$ & $10(34)$ & \multirow[t]{2}{*}{0,124} & 11,2 & $40(41)$ \\
\hline & Incorreto & $27(39)$ & $5(17)$ & & 8,7 & $32(33)$ \\
\hline & Não sabe ou não respondeu & $12(17)$ & $14(48)$ & \multirow{3}{*}{0,483} & 11,0 & $26(27)$ \\
\hline \multirow{4}{*}{$\begin{array}{l}\text { Realização de cuidados ao recém-nascido e sua família baseados no } \\
\text { Método Canguru }\end{array}$} & Já realizou cuidados do MC & $53(77)$ & $11(38)$ & & 10,5 & $64(65)$ \\
\hline & Nunca realizou cuidados do & & & & & \\
\hline & $\mathrm{MC}$ & $15(22)$ & $14(48)$ & \multirow{4}{*}{0,564} & 12,4 & $29(30)$ \\
\hline & Não sabe ou não respondeu & $1(1)$ & $4(14)$ & & 11,8 & $5(5)$ \\
\hline \multirow{2}{*}{$\begin{array}{l}\text { Conhecimento dos critérios para que o recém-nascido de baixo peso vá } \\
\text { para a Unidade de Cuidados Neonatal Intermediário Canguru }\end{array}$} & Conhece os critérios & $59(86)$ & $13(45)$ & & 11,0 & $72(73)$ \\
\hline & Não conhece ou não respondeu & $10(14)$ & $16(55)$ & & 11,4 & $26(27)$ \\
\hline
\end{tabular}

Fonte: Elaboração própria 
Tabela 3 - Conhecimentos dos profissionais da saúde da linha pediátrica sobre o Método Canguru em função do setor de atuação. Campo Grande-MS, 2020/2021.

Conhecimentos dos profissionais da saúde da linha pediátrica sobre o método canguru

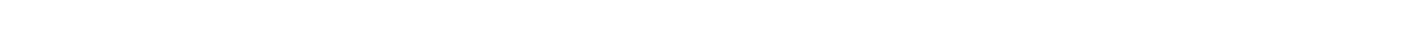

BLH

\section{UTI}

Setores

PEDIATRIA PEDIATRICA

Tem conhecimento

$4 \quad(80)$

n $(\%)$

n $(\%)$

n $(\%)$

Conhecimento sobre o número de etapas do Método Canguru

Não tem conhecimento

1 (20)

$47 \quad(90)$

9 (41)

5 (10)

3 (14)

$10(53)$

sabe ou não

respondeu

$0 \quad(0)$

Conhecimento sobre o momento correto de se iniciar o Método

Tem conhecimento

$0 \quad(0)$

$8 \quad(42)$

\section{Canguru}

Conhecimento sobre tempo recomendado para a realização da
Posição Canguru

Não tem conhecimento

3 (60)

Correto

$2(40)$

Incorreto

$0 \quad(0)$

36 (69)

$10 \quad(45)$
$11 \quad(50)$

$8 \quad(42)$

Posição Canguru

Não sabe ou não

respondeu

$0 \quad(0)$

$16 \quad(31)$

$11 \quad(50)$

$11 \quad(58)$

Já realizou cuidados do

MC

$\begin{array}{rr}3 & (14) \\ 19 & (86)\end{array}$

8 (42)

Realização de cuidados ao recém-nascido e sua família baseados no Método Canguru

do $\mathrm{MC}$

$0 \quad(0)$

$46+(88)$

$0 \quad(0)$

Não sabe ou não respondeu

Conhecimento dos critérios para o recém-nascido de baixo peso

Conhece os critérios

ir para a Unidade de Cuidados Neonatal Intermediário

Não conhece ou não

Canguru

respondeu

$5 \quad(100)$

$6 \quad(12)$

$7 \quad(32)$

$11(58)$

Fonte: Elaboração própria

$2(40)$

$49 \quad(94)$

$11 \quad(50)$

$7 \quad$ (37) 


\section{DISCUSSÃO}

Os participantes eram em sua maioria mulheres, técnicas de enfermagem, com mais de 40 anos de idade, pós-graduadas e com tempo de serviço na instituição de aproximadamente 12 anos. A pesquisa demonstrou um maior percentual de respostas adequadas sobre o método, a PC e sobre os seus benefícios nas respostas dos profissionais da saúde que tinham o curso do MC, mais tempo de serviço na linha pediátrica e que atuavam na unidade neonatal. Além disso, a maioria dos participantes tem conhecimento sobre o método e em quantas etapas se divide. Entretanto, alguns participantes têm a opinião equivocada de que o MC se restringe ao contato pele a pele da mãe/pai com o bebê.

O MC é uma mudança de paradigma da atenção perinatal, na qual a humanização e a qualidade do atendimento e do cuidado oferecido constituem objetivos fundamentais, além de, um compromisso de todo $\mathrm{o}$ profissional de saúde com o RNPTBP e sua família ${ }^{(3)}$. Ou seja, o método envolve a diminuição de estímulos ambientais, o controle da dor, facilita a interação do $\mathrm{RN}$ com a família, orientação e o suporte ao aleitamento materno e a PC. Desta maneira, o contato pele a pele, que consiste em manter o $\mathrm{RN}$, somente de fraldas, na posição vertical junto ao peito dos pais com o apoio, orientação e acompanhamento do profissional da saúde é um dos cuidados do MC ${ }^{(5,9)}$. No entanto, neste estudo, muitos profissionais de saúde relacionaram o MC apenas ao contato pele a pele e desconhecem a abrangência do método.

Estudo realizado com 37 profissionais de enfermagem da UTIN de um hospital paranaense revelou que os participantes não relacionavam a assistência de saúde prestada aos $\mathrm{RN}$ com a prática do $\mathrm{MC}$, pois $\mathrm{o}$ entendiam como rotina de trabalho, o que demonstra a necessidade de apropriação do MC com sua aplicabilidade diária (10). Já, pesquisa com abordagem qualitativa envolvendo oito enfermeiras da linha materno-infantil de um hospital de Minas Gerais (MG), Brasil, demonstrou que três delas conheciam o MC na teoria, mas não tiveram a oportunidade de trabalhar com ele na prática (11). Outro estudo, também qualitativo, com 15 profissionais da enfermagem que atuavam em uma UTIN de Porto Alegre (RS), apontou que os profissionais apresentam o MC como uma prática para garantir a humanização do $\mathrm{RN}$ e fortalecer o vínculo com seus familiares ${ }^{(12)}$. Assim como, nesta investigação, que demonstrou o desconhecimento de alguns participantes em relação ao $\mathrm{MC}$, as suas etapas, que abrange o pré-natal, o nascimento, a hospitalização do RNPTBP na UTIN e UCINCo e não apenas na UCINCa e as consultas de retorno até o peso de 2.500 gramas.

A PC traz inúmeros benefícios tanto para o RNPTBP quanto para os pais. Um trabalho realizado com 86 neonatos de um hospital em Rondônia (RO) mostrou que 
aqueles submetidos ao contato pele a pele com seus pais apresentaram maior ganho de peso e menor tempo de internação quando comparados aos RN nos quais a PC não foi aplicada ${ }^{(13)}$. Os benefícios do contato pele a pele ao RNPTBP são os mesmos, seja realizado pela mãe, seja pelo pai ${ }^{(14)}$. Fato que corrobora com os achados nesta investigação que apontou que a maioria dos participantes responderam que a PC é o "Contato pele a pele do RNPTBP com seus pais, junto ao peito na posição vertical".

Vários benefícios do MC são apontados pelos profissionais da saúde da atual pesquisa e todos aprovam o método. Os três benefícios mais mencionados foram: "Aumenta o vínculo familiar e bebê", "Estimula/facilita a amamentação" e "Favorece o desenvolvimento neuropsicomotor do RN". Estudo observacional desenvolvido por meio de filmagens evidenciou que o maior tempo de PC beneficia as trocas iniciais de contato entre o filho pré-termo e a mãe, o que sugere maior estado de alerta e melhor disponibilidade do RN para interações com a mãe durante a amamentação ${ }^{(15)}$. Pesquisa realizada com 37 profissionais da enfermagem demostrou que $33,8 \%$ dos profissionais associam o MC a uma melhora no crescimento e desenvolvimento do $\mathrm{RN}^{(16)}$.

Investigação quase experimental realizada em dois hospitais da Turquia, cujo objetivo era investigar o efeito do MC na taxa de amamentação e no desenvolvimento de
RNPTBP nos primeiros seis meses de vida evidenciou que o grupo que realizou a PC teve uma média de peso corporal significativamente maior do que o grupo controle no terceiro e sexto mês pós-natal (p $<0,05)$. Além disso, o grupo experimental teve ingestão de leite materno e taxas de amamentação maiores do que o grupo controle durante a transição para a amamentação exclusiva e na alta, e no primeiro, terceiro e sexto mês pós-natal ( $\mathrm{p}$ $<0,05)^{(17)}$.

Ademais, $\quad 66$ profissionais argumentaram que o MC beneficia o RN e 52 disseram que o método beneficia a mãe. No entanto, o MC não beneficia somente o RN, mas também seus pais, familiares, irmãos e os profissionais da saúde, uma vez que reduz o tempo de separação mãe/pai-filho e facilita o vínculo. O RN se beneficia com a redução de infecção hospitalar, estresse e dor, a melhora do controle térmico e neuropsicomotor, e um melhor relacionamento e comunicação entre a família e os profissionais da saúde. Outra vantagem é que os irmãos podem vir às unidades de saúde para ver o bebê. ${ }^{(5)}$ Além de que, quando a equipe multiprofissional percebe a melhora clínica e a evolução do RNPTBP após a realização da PC, os mesmos passam a ter credibilidade no método, notam que não é dogma e sim ciência, e assim, esta prática continua sendo adotada nas unidades neonatais ${ }^{(18)}$.

Pesquisa internacional realizada com 27 profissionais de um hospital terciário e três 
hospitais distritais do Malawi evidenciou que na percepção dos participantes os bebês do alojamento canguru por serem mais estáveis possuem uma baixa priorização de atendimento e práticas inadequadas de monitoramento ${ }^{(19)}$. Outra investigação também desenvolvida no Malawi com 123 profissionais de saúde revelou reações positivas destes em relação aos cuidados desenvolvidos pelos familiares aos bebês prétermo durante a hospitalização, assim como, ao contato pele a pele ${ }^{(20)}$. Fato que corrobora com os achados deste estudo no qual, os profissionais disseram prestar os cuidados centrados no RN e sua família. No entanto, deve-se ressaltar que a participação dos pais não deve interferir na qualidade do atendimento prestado pelos profissionais de saúde ao $\mathrm{RN}$, pelo contrário a inclusão dos pais só tem a contribuir.

No tocante aos cuidados relacionados ao MC aplicados pelos profissionais em ambiente hospitalar, o apontado com mais frequência pelos participantes foi: "Orientações e acolhimento aos pais sobre os cuidados ao RN". Em concordância com o resultado desta pesquisa, um estudo qualitativo com profissionais da enfermagem de uma UCINCa no Nordeste brasileiro destacou a importância das orientações sobre o MC aos pais/familiares, o que favorece a adesão deles ao método ${ }^{(21)}$. Além disso, ao acolherem as famílias de RNPTBP mediando o primeiro contato dos pais com seus filhos, os profissionais de saúde estão proporcionando a formação do vínculo entre eles interrompida por causa de um parto prematuro e a necessidade de hospitalização do filho na unidade neonatal ${ }^{(5)}$.

Além do mais, o contato pele a pele deve ser iniciado o quanto antes e durante a hospitalização do RNPTBP na UTIN e UCINCo. Assim, investigação realizada em um hospital universitário em Gâmbia, cujo objetivo era compreender os efeitos dos cuidados iniciais com o MC na sobrevivência de RN instáveis e com menos de $2.000 \mathrm{~g}$ evidenciou redução da mortalidade pela metade durante o período de implementação do estudo e a importância de intervenções relacionadas ao $\mathrm{MC}$ em neonatos instáveis em unidades neonatais ${ }^{(22)}$.

Neste estudo, somente dois profissionais de saúde relataram ter o curso de tutor do MC hospitalar, ou seja, com licença para ser um replicador e realizar as capacitações. Tal fato demostra a necessidade de formação de novos tutores visto que o hospital é a referência do Estado e o responsável pelas capacitações internas e externas, além de promover a sensibilização da equipe de saúde para o MC.

Dessa maneira, a aquisição de conhecimento é fundamental para o desenvolvimento e a implementação de boas práticas do método nas unidades de saúde. $\mathrm{O}$ conhecimento teórico precisa estar articulado com a mudança comportamental da equipe, trabalhando na elaboração de estratégias gerenciais que facilitem a adesão ao MC na 
prática assistencial ${ }^{(23)}$. Assim, os cursos do MC habilitam os profissionais para que eles adquiram base científica para as suas condutas e melhorem a assistência prestada ao RNPTBP e suas famílias.

Estudo qualitativo realizado no Quênia, cujo objetivo era compreender do ponto de vista dos familiares e do profissional de saúde, as barreiras e os facilitadores para os cuidados essenciais ao $\mathrm{RN}$, demonstrou que o MC é amplamente aceito como uma intervenção padrão ouro para aumentar a sobrevivência de RNPTBP. Além disso, esta pesquisa incluiu os pontos de vista e opiniões dos pais, familiares, profissionais de saúde, legisladores e representantes não governamentais no contexto da prestação de cuidados ao $\mathrm{RN}$ e o desenvolvimento de um programa de trabalho mais amplo de desenvolvimento de uma pontuação de alerta precoce. Demonstrando a importância do envolvimento de todos para que ocorra a implementação efetiva do MC ${ }^{(24)}$.

Alguns fatores podem ser mencionados como limitações para a efetivação de outras análises, reflexões e apontamentos. Um deles se refere ao questionário, considerado longo e complexo por alguns profissionais, o que não permitiu as suas adesões à pesquisa; o outro é que o hospital em pauta é o único do estado com os títulos de MC e IHAC, o que não reflete a realidade de todos os hospitais. Ainda mais importante, trata-se de um momento atípico vivenciado por todo o mundo, que são as limitações trazidas pela pandemia, o que fez com que diversos profissionais fossem realocados da linha pediátrica para outros setores a fim de prestarem cuidados para pacientes de diversas faixas etárias.

\section{CONCLUSÕES}

Este estudo demonstrou que alguns participantes tem o conhecimento teórico sobre o MC, sabe em quantas etapas se divide e seus principais benefícios. Além de que, a maioria dos profissionais realizara o curso de MC pelo hospital do estudo. Entretanto, as questões abertas revelaram que alguns participantes têm a opinião equivocada de que o MC é somente o contato pele a pele da mãe/pai com o bebê, ao passo que a metodologia canguru é ampla e envolve uma mudança de paradigma como a importância do aleitamento materno exclusivo (AME), a assistência humanizada e a inserção da família nos cuidados. Por outro lado, os profissionais souberam descrever vários benefícios do método condizentes com as diretrizes e estudos científicos sobre tal, principalmente os participantes que tinham capacitação, isto é, a pesquisa mostrou que existe diferença estatística entre os profissionais que realizaram o curso e os que não o realizaram, ao serem questionados sobre quem se beneficiava com o MC.

A investigação possibilitou a reflexão sobre o entendimento do MC pelos profissionais da saúde que realizam 
assistência aos RNPTBP e seus familiares. Tal fato pode contribuir para $\mathrm{O}$ aperfeiçoamento assistencial, além de incentivar os gestores de saúde na inserção da educação permanente e continuada dos profissionais da saúde sobre o MC. Este estudo é importante para a enfermagem também, visto que o enfermeiro favorece a formação do vínculo bebê-pais-profissionais e deve ter uma escuta qualificada, incluindo os pais nos cuidados aos neonatos $\mathrm{e}$ proporcionando atendimento interdisciplinar e humanizado. A partir dos dados desta pesquisa, fica clara a necessidade da realização de novos estudos sobre a percepção e aplicação do MC aos RNPT e sua família pela equipe multiprofissional de saúde, tanto em hospitais públicos quanto em privados.

\section{REFERÊNCIAS}

1. Barraza PCG, Jofré AVE, Ramírez OJA. Percepciones Asociadas al Método Madre Canguro de Madres con Recién Nacidos Prematuros. Cienc enferm [Internet]. 2020; 26:32-2.

2. Lopes BA, Oliveira ALCB, Costa GR, Costa AA, Moraes LMV, Maia JM, Bezerra MAR. Características maternas e dos recémnascidos admitidos em uma unidade de terapia intensiva neonatal. Rev Enferm Atual in Derme. 2020; 93(31):e-020022.

3. Oliveira ND, et al. Atenção Humanizada ao Recém-nascido de Baixo Peso - Método Canguru: 15 anos de uma política pública de saúde que mudou o cuidado perinatal brasileiro. In: Cera SMT, Azevedo CR, Oliveira VMG, Streit MD, Carvalho LZ. Método Canguru no Brasil: 15 anos de política pública. São Paulo (SP): Instituto de Saúde. 2015 [citado 2020 Abril
13].

Disponível

em: https://portaldeboaspraticas.iff.fiocruz.br/wpcontent/uploads/2017/10/canguru_capa_miolo .pdf.

4. Brasil. Ministério da Saúde. Secretaria de Atenção à Saúde. Departamento de Ações Programáticas Estratégicas. Método canguru: diretrizes do cuidado. Brasília (DF): Ministério da Saúde; 2018. Disponível em: http://portaldeboaspraticas.iff.fiocruz.br/wpcontent/uploads/2018/09/metodo_canguru_dir etrizes_cuidado2018.pdf.

5. Brasil. Ministério da Saúde. Secretaria de Atenção à Saúde. Departamento de Ações Programáticas Estratégicas. Atenção humanizada ao recém-nascido: Método Canguru: manual técnico. 3 ed. Brasília (DF): Ministério da Saúde; 2017. Disponível em: http://bvsms.saude.gov.br/bvs/publicacoes/ate ncao_humanizada_metodo_canguru_manual_ 3ed.pdf.

6. Souza JR, Ribeiro LM, Vieira GB, et al. Método Canguru na Perspectiva dos Profissionais de Saúde de uma Unidade de Neonatologia. Enferm em Foco, 2019; 10(2):1604

7. Gesteira E, Braga P, Nagata M, Santos L, Hobl C, Ribeiro B. Método canguru: benefícios e desafios experienciados por profissionais de saúde. Rev Enferm UFSM. 2017;6(4):518-28.

8. Aires LCP, Kock C, Santos EKA, et al. Kangaroo-mother care method: a documentary study of theses and dissertations of the brazilian nurse (2000-2017). Rev Bras Enferm. 2020;73(2):e20180598.

9. Alves FN, Azevedo VMGO, Moura MRS, Ferreira DMLM, Araújo CGA, Mendes-Rodrigues C, Wolkers PCB. Impacto do método canguru sobre o aleitamento materno de recém-nascidos pré-termo no Brasil: uma revisão integrativa. Cien Saúde Col. 2018; 25(11): 4509-20.

10. Stelmak AP, Freire MHS. Aplicabilidade das ações preconizadas pelo método canguru. Rev Cuid Fund On-line. 2017;9(3):795-802. 
11. Ferreira DO, Silva MPC, Galon T, Goulart BF, Amaral JB, Contim D. Método canguru: percepções sobre o conhecimento, potencialidades e barreiras entre enfermeiras. Esc Anna Nery. 2019;23(4):e20190100.

12. Mantelli GV, Strapasson MR, Pierotto AA, Renosto JM, Silva JF. Método canguru: percepções da equipe de enfermagem em terapia intensiva neonatal. Rev Enferm UFSM. 2017;7(1):51-60.

13. Souza AKCM, Tavares ACM, Carvalho DGL, Araújo VC. Ganho de peso em recém-nascidos submetidos ao contato pele a pele. Rev CEFAC. 2018;20(1):53-60.

14. Martins ASD, Nascimento JDB, Santos ÍR, Silva JSL, Rêgo RMV, Andrade ASA, Galloti FCM. Participação do pai no método canguru: conhecimentos, dificuldades, motivações e sentimentos. Cien Biol Saúde Unit. 2019;5(2):95-105.

15. Nascimento NCR, Campos LG, Lucena AM, Pereira JM, Costa PR, Lima FAF, Azevedo VMGO. Relação da duração da posição canguru e interação mãe-filho prétermo na alta hospitalar. Rev Paul Ped. 2017;35(2):136-43.

16. Stelmak AP, Mazza VA, Freire MHS. $\mathrm{O}$ valor atribuído pelos profissionais de enfermagem aos cuidados preconizados pelo método canguru. Rev Enferm UFPE on-line. 2017;11(9):3376-85.

17. Kucukoglu S, Yilmaz KF, Aytekin OA, Ozcan Z. The effect of kangaroo care on breastfeeding and development in preterm neonates. J Pediatr Nurs. 2021;21: S08825963.

18. Gianini, NOM. Trajetória do Método Canguru nas Unidades da Secretaria Municipal de Saúde do Rio de Janeiro. In: Cera SMT, Azevedo CR, Oliveira VMG, Streit MD, Carvalho LZ. Método Canguru no Brasil: 15 anos de política pública. São Paulo (SP): Instituto de Saúde. 2015 Disponível em:

https://portaldeboaspraticas.iff.fiocruz.br/wpcontent/uploads/2017/10/canguru_capa_miolo .pdf.
19. Kinshella MW, Salimu S, Chiwaya B, et al. "So sometimes, it looks like it's a neglected ward": Health worker perspectives on implementing kangaroo mother care in southern Malawi. PLoS ONE, 2020; 15(12):e0243770.

20. Mhango P, Chipeta E, Muula AS, et al. Implementing the Family-Led Care model for preterm and low birth weight newborns in Malawi: Experience of healthcare workers. Afr J Prim Health Care Fam Med. 2020; 12(1): e1-11.

21. Sales IMM, Santos JDM, Rocha SS, Gouveia MTO, Carvalho NAR. Contribuições da equipe enfermagem na segunda etapa do Método Canguru: Implicações para a alta hospitalar do recém-nascido. Esc Anna Nery. 2018;22(4):e20180149.

22. Brotherton H, Gai A, Kebbeh B, et al. Impact of early kangaroo mother care versus standard care on survival of mild-moderately unstable neonates <2000 grams: A randomised controlled trial. EClinicalMedicine, 2021; 39:101050.

23. Silva LJ, Leite JL, Silva TP, Silva ÍR, Mourão PP, Gomes TM. Desafios gerenciais para boas práticas do Método Canguru na UTI Neonatal. Rev Bras Enferm. 2018;71(6):2783-91.

24. Mitchell EJ, Pallotti P, Qureshi ZP, Daniels JP, Oliver M, Were F, Osoti A, Gwako G, Kimani V, Opira J, Ojha S. Parents, healthcare professionals and other stakeholders' experiences of caring for babies born too soon in a low-resource setting: a qualitative study of essential newborn care for preterm infants in Kenya. BMJ Open. 2021;11(6):e043802.

\section{Autor correspondente}

Mayara Carolina Cañedo, Rua: Casimiro de Abreu n. 135. Jardim dos Cristhais, Dourados - MS, CEP 79821026, +55 (67) 99177-2636. E-mail: maycarolina@ hotmail.com

Submissão: 2021-09-19

Aprovado: 2021-12-05 